\title{
Visionen zum Schaden des Schweizer Gesundheitswesens
}

\author{
Bruno Henggi \\ Verantwortlicher Public Affairs
}

Der Politik in Bern wird oft vorgehalten, es fehle an Visionen. Es wäre indessen unzulässig, diesen Vorwurf dem politisch verantwortlichen Bundesrat für das Kostendämpfungspaket II zu machen. Patienten bevormunden, Leistungserbringer dirigieren und Tarifpartner übersteuern: Mit zentralistischen Eingriffen in die Bewegungsfreiheit der Patienten und Versicherten und in den Gestaltungsspielraum der Akteure im Gesundheitswesen soll der Weg hin zu einer staatlich gelenkten medizinischen Versorgung in der Schweiz geebnet werden.

Es scheint schwer vorstellbar, dass die Patienten hierzulande auf dem Weg zum Arzt der eigenen Wahl zuerst eine Konsultation bei einer Beratungsstelle vornehmen wollen. Denn die freie Arztwahl ist ein Fixpunkt in den Wertvorstellungen der Schweizer Bevölkerung. Heute können die Versicherten frei wählen, von welchem Leistungserbringer sie sich behandeln lassen wollen. Sie können aber auch eine Versicherung mit eingeschränkter Wahl abschliessen. 2018 wählten rund 70 Prozent der Versicherten ein solches Versicherungsmodell. Neu sollen alle Schweizerinnen und Schweizer zuerst eine Beratungsstelle besuchen müssen, wenn sie medizinische Leistungen in Anspruch nehmen wollen. Dies unabhängig davon, wie sie versichert sind. Gemäss den Vorstellungen der Visionäre im Departement des Innern wird eine Patientin ihre Erstberatungsstelle aus der kantonalen Liste der Erstberater auswählen. Sie holt bei der Erstberatungsstelle die Bestätigung ein, dass diese bereit ist, diese Rolle für sie zu übernehmen. Die Bestätigung muss sie anschliessend beim Versicherer einreichen. Sollte die Patientin aus welchem Grund auch immer die Erstberatung wechseln wollen, wird sie dies dann tun können, wenn die Voraussetzungen gegeben sind, welche der Bundesrat bestimmt. Letzterer bestimmt auch, unter welchen Voraussetzungen eine Versicherung für einen Versicherten die Erstberatung bestimmen kann.

\section{Erstberatung bedeutet Zwangsberatung für alle}

Erstberater sind gemäss der bundesrätlichen Vorstellung Hausärzte, Gruppenpraxen, telemedizinische Zen- tren oder Netzwerke zur koordinierten Versorgung. Für Ärztinnen und Ärzte werden an Weiterbildungstiteln Allgemeine Innere Medizin, Kinder- und Jugendmedizin, praktischer Arzt/praktische Ärztin verlangt. Sie gelangen auf die kantonale Liste der Erstberatungsstellen, indem sie dem Kanton ein entsprechendes Gesuch vorlegen. Der Kanton wird eine Verfügung erlassen und dem Leistungserbringer Bescheid geben, ob dem Gesuch entsprochen wird. Der Bundesrat hat die Absicht, auch die Leistungen der Erstberatung zu bestimmen und sie pauschal zu vergüten. Er wird die Höhe der jährlichen Pauschale nach Anhörung der Tarifpartner festlegen, welche die Erstberatungsstelle pro Patienten er-

Der Bundesrat hat die Absicht, auch die Leistungen der Erstberatung zu bestimmen und sie pauschal zu vergüten.

hält, und er wird die Leistungen bezeichnen, welche durch die Pauschale als abgedeckt gelten. Die Pauschalen werden dem Leistungserbringer unabhängig von der Inanspruchnahme der Erstberatung ausgerichtet.

Wer sich beruflich dafür einsetzt, den Bedürfnissen der Patienten gerecht zu werden und ihnen die bestmögliche medizinische Versorgung anzubieten, kann eine pauschale Abgeltung einer Erstbeurteilung nicht gutheissen. Ebenso wenig kann er unterstützen, dass die Freiwilligkeit bei der Entscheidung für ein Versicherungsmodell mit eingeschränkter Wahl durch ein Obligatorium abgelöst werden wird.

Ein Patient oder eine Patientin, welche mit einer Allergie oder einem Ekzem konfrontiert ist, wird gerne ohne Zeitverzug den medizinischen Rat der Dermato- 
login in Anspruch nehmen wollen. Den obligatorischen Gang zur Erstberatung wird man als Verschlechterung der Versorgung erleben. Und es ist darüber hinaus anzunehmen, dass wegen der zusätzlichen Zwangskonsultation höhere Kosten entstehen werden.

\section{Zielvorgabe: Die einschneidendste Massnahme der Kostendämpfungspolitik des EDI}

Die Zielvorgabe ist jenes Element im Kostendämpfungspaket, das die einschneidendsten Folgen für das Gesundheitswesen haben kann. Mit diesem Instrument wird in die bestehenden Zuständigkeiten eingegriffen und das Machtgefüge zugunsten der Kompe-

Immerhin ist sich das Departement des Innern bewusst, dass es ein administratives Ungetüm schafft.

tenzen des Bundes verschoben. Die Zielvorgabe basiert auf der Prämisse, dass die Verwaltung über die Planungskompetenz verfügt, am richtigen Ort Kosten zu sparen. Ausserdem scheint die Verwaltung davon auszugehen, dass ihre Behauptung, wonach 20 Prozent der Kosten ohne Qualitätseinbussen eingespart werden können, auf Fakten basiert und deshalb die Zielvorgabe - jedenfalls in der Anfangsphase - ohne Qualitätseinbussen umgesetzt werden kann. Immerhin ist sich das Departement des Innern bewusst, dass es ein administratives Ungetüm schafft. Im erläuternden Bericht des Bundesrates wird von initialem Vollzugsaufwand und von administrativem Aufwand für Bund, Kantone und Tarifpartner gesprochen. Eine Machbarkeitsstudie hat das EDI jedoch nicht in Auftrag gegeben. Im Wesentlichen will der Bund ein Gesamtkostenziel definieren, den Kantonen Empfehlungen abgeben zur Festsetzung des je individuellen Kostenziels pro Kanton sowie auch Ratschläge erteilen über die Aufteilung des Wachstums auf die Kostenblöcke. Den Kantonen obliegt es, über ihre je eigenen Kostenziele zu entscheiden und das Wachstum über kantonale Vorgaben auf die Kostenblöcke zu verteilen und allenfalls die Aufteilung noch weiter zu spezifizieren und zum Beispiel verschiedene Wachstumsziele für Grundversorger und Spezialisten zu definieren. Korrekturmassnahmen, ob freiwillig oder verbindlich, sollen aus Anpassungen der Tarifverträge bestehen. Zur weiteren Schwächung der Tarifpartnerschaft werden hier subsidiäre Kompetenzen von Bund und Kantonen zum Zuge kommen. Die Chancen, dass sich die Tarifpartner auf ungenügende Tarifierungen einigen, ist ziemlich gering. Deshalb sollen die Kantone selbst Massnahmen verfügen können oder müssen. Dabei wird es um Änderungen der Tarife und Preise gehen (Taxpunktwerte, Baserates). Dass mit sanktionierbaren Zielvorgaben das Versicherungssystem ausgehebelt werden könnte, wird vom Bundesrat im erläuternden Bericht mit wenigen Zeilen negiert. Die Verfassungskonformität scheint ihn nicht sonderlich zu interessieren. Die Frage der Verfassungsmässigkeit der Pläne des Gesundheitsministers würde eine eingehende Prüfung verdienen.

Im Rahmen der Vernehmlassung besteht dafür Gelegenheit. Sie dauert bis zum 19. November. Mit der Botschaft ist rund ein halbes Jahr später zu rechnen.

\section{Die Umsetzung der Zielvorgabe ist bereits im Parlament}

Eine Umsetzungsmassnahme der Zielvorgabe ist bereits in der parlamentarischen Beratung. Es handelt sich um die Massnahmen der Tarifpartner zur Kostensteuerung. Diese Massnahme war in der Kommission umstritten, wurde aber im Teil 2 des Kostendämpfungspakets 1 beschlossen und wird in der Sondersession Ende Oktober im Nationalrat beraten. Diese neue

Die neue gesetzliche Vorgabe soll die Tarifpartner dazu anhalten, beispielsweise degressive Tarife zu vereinbaren.

gesetzliche Vorgabe soll die Tarifpartner dazu anhalten, beispielsweise degressive Tarife zu vereinbaren. Auch hier wäre die Verfassungsmässigkeit zu prüfen. Sollten bei Nichteinigung der Tarifpartner ungenügende Tarife durch den Staat festgesetzt werden, wäre dies gemäss bundesgerichtlicher Rechtsprechung eine Verletzung des Versicherungsprinzips. Denn die Tarifierung ist gegenüber dem Leistungsanspruch nachrangig.

Die übrigen Massnahmen des Kostendämpfungspakets 1, welche für die Ärzteschaft von direktem Interesse sind, betreffen im Wesentlichen die Frage, wie viel mehr Einfluss dem Bund im Bereich der Tariforganisation, der Tarife und der Daten zugestanden werden soll. Während der Nationalrat dem Machthunger des Departements des Innern grosse Zugeständnisse machte, ist zumindest die Ständeratskommission zurückhaltender. 